G. P. Wever

Nagoya Math. J.

Vol. 84 (1981), 9-30

\title{
THE MODULI OF A CLASS OF RANK 2 VECTOR BUNDLES ON $P^{3}$
}

\author{
G. PETE WEVER
}

\section{Introduction}

Barth and others [1], [2], [5] have begun the study of stable algebraic vector bundles of rank 2 on projective space. Maruyama [7] has shown that stable rank 2 bundles have a variety of moduli which is the finite union of quasi-projective varieties.

The point of view taken here is to study a rank 2 vector bundle on $\boldsymbol{P}=\boldsymbol{P}_{k}^{3}, k$ an algebraically closed field, by looking at the zero sets of the global sections of the bundle. The zero set is a curve in $P$. A skew bundle on $P$ is a rank 2 bundle which has a global section whose zero set is a pair of skew lines. Skew bundles are precisely the stable rank 2 bundles with Chern classes (which we consider as integers) $c_{1}=c_{2}=2$. This paper gives the explicit description of the moduli of skew bundles as an open subscheme in $\boldsymbol{P}_{k}^{5}$. These bundles, see Remark 2.1.3, were among the first known examples of indecomposable vector bundles on $P[6$, Section 10]. From a point of view different from the present one Barth [1, Section 7] has implicitly given the moduli of these bundles which he calls null-correlation bundles.

More precisely we do the following. A skew bundle parametrized by a scheme $T$ is a rank 2 vector bundle $E$ on $P_{T}^{3}$ such that the restrictions of $E$ to the fibres over geometric points of $T$ are skew bundles. For a locally noetherian scheme $T$ let $S k(T)=\{E \mid E$ skew bundle parametrized by $T\} / \sim$, where $E_{1} \sim E_{2}$ if there exists some isomorphism of $E_{2}$ and $E_{1} \otimes_{P_{T}^{3}} L$ where $L$ is the pullback of a line bundle on $T$. If $g: T^{\prime} \rightarrow T$ is a morphism and $E$ a skew bundle parametrized by $T$ then the pullback of $E$ to $P_{T^{\prime}}^{3}$ is a skew bundle parametrized by $T^{\prime}$. This gives a natural map $g^{*}: S k(T)$ $\rightarrow S k\left(T^{\prime}\right)$. Clearly $S k$ is a contravariant functor from the category of locally noetherian schemes to the category of sets. Our main theorem (Theorem

Received October 3, 1979. 
3.5) says that $S k$ is a representable functor. That is, there is a scheme $M$ and a class of skew bundles parametrized by $M$ which defines in the obvious way a morphism of functors $\Phi: \operatorname{Hom}(, M) \rightarrow S k$ and $\Phi$ is an isomorphism. $M$ is called the fine moduli for skew bundles. The construction in this paper arises naturally from the geometry and is independent of the techniques of [7], [8].

In Section 1 we establish the basic techniques and give some preliminary results. In Section 2 we give a method for constructing families of skew bundles. In Section 3 we show that there is a fine moduli for skew bundles. The moduli has a natural compactification and in Section 4 we show that geometric points of the compactification correspond to stable coherent sheaves on $P$.

Notations and Conventions

All schemes are assumed to be locally noetherian. If $F$ is a coherent sheaf on a scheme $T$ let $F^{\vee}$ denote the dual $\mathscr{H}_{o m}\left(F, \mathcal{O}_{T}\right)$. If $t$ is a point of $T$ then $k(t)$ denotes the residue class field $\mathcal{O}_{t, T} / m_{t}$. If $f: X \rightarrow T$ is a morphism of schemes then $X_{t}=X \times_{T}$ spec $k(t)$ is the scheme fibre of $f$ over $t$ and if $F$ is a sheaf on $X$ then $F_{t}$ is the restriction of $F$ to the fibre $X_{t}$. A geometric point of $T$ is a morphism spec $K \rightarrow T, K$ an algebraically closed field. By abuse of notation we will denote this morphism by $t$ and $K$ by $k(t)$ and say $t$ is a geometric point of $T$. With the morphism $f$ above, we define the geometric fibre $X_{t}$ of $f$ over the geometric point $t$ and the restriction $F_{t}$ of $F$ to the geometric fibre. It will be clear from the context whether we are referring to a usual point or a geometric point of a scheme. If $E$ is a sheaf on $T$ and $t$ is either a usual point or a geometric point of $T$ then the fibre of $E$ at $t$ is the restriction of $E$ to $\operatorname{spec} k(t)$ and is denoted by $E(t)$.

I would like to thank Arthur Ogus and Robin Hartshorne for generously sharing their time and enthusiasm with me as I did this work.

\section{§1. Preliminaries}

In this section $k$ denotes an algebraically closed field and $P=\boldsymbol{P}_{k}^{3}$. Our basic technique for studying vector bundles (locally free sheaves) is the correspondence between rank 2 bundles $E$ and curves $Y$ which arise as the zero sets of the global sections. If $s \in H^{\circ}(P, E)$ then $Y=Z(s)$ is the scheme zero set of $s$. If $Y$ is nonempty and has codimension $\geq 2$ then $Y$ is in fact a local complete intersection curve and $s$ defines an exact sequence 


$$
0 \longrightarrow \mathcal{O}_{P} \longrightarrow E \longrightarrow \mathscr{I}_{Y}\left(c_{1}\right) \longrightarrow 0 \text {. }
$$

Conversely, given the curve $Y$ the bundle can be recovered as an extension. For details and proofs of this and the following see [5, Sections 1, 2]. For a curve $Y$ in $P, \omega_{Y}=\mathscr{E} x t_{P}^{2}\left(\mathcal{O}_{Y}, \omega_{P}\right)$ is the dualizing sheaf. The precise statement of the correspondence is as follows.

1.0.2. A local complete intersection curve $Y$ in $P=\boldsymbol{P}_{k}^{3}$ occurs as the scheme zero set of a rank 2 vector bundle if and only if $\omega_{Y}$ is isomorphic to the restriction of a line bundle from $P$. In this case $\omega_{Y} \cong \mathcal{O}_{Y}\left(c_{1}-4\right)$ where $c_{1}$ is the first Chern class of the bundle.

1.0.3. If $Y$ is a curve which comes from a rank 2 bundle with Chern classes $c_{1}$ and $c_{2}$ then the degree of $Y$ is $d=c_{2}$ and arithmetic genus is $P_{a}=\left(c_{2}\left(c_{1}-4\right) / 2\right)+1$.

Let $Y$ be the union of two skew lines $L$ and $L^{\prime}$. We have $\mathcal{O}_{Y}=\mathcal{O}_{L}$ $\oplus \mathcal{O}_{L^{\prime}}, \omega_{Y}=\mathcal{O}_{Y}(-2)$ and $P_{a} Y=-1$. By the preceding $Y$ comes from a vector bundle $E$ which has Chern classes $c_{1}=c_{2}=2$. For a coherent sheaf $F$ let $h^{i}(P, F)$ denote $\operatorname{dim}_{k} H^{i}(P, F)$.

Proposition 1.1. Let $Y$ be two skew lines $L$ and $L^{\prime}$. Then the homogeneous ideal of $Y$ is generated by 4 quadrics and $h^{1}\left(P, \mathscr{I}_{Y}(n)\right)=0$ for $n$ $\neq 0$ and $h^{1}\left(P, \mathscr{I}_{Y}\right)=1$.

Proof. By a suitable choice of homogeneous coordinates we may suppose $I_{L}=(x, y)$ and $I_{L^{\prime}}=(z, w)$. Then $I_{Y}=(x z, x w, y z, y w)$ is generated by 4 quadrics. From the standard exact sequence

$$
0 \longrightarrow \mathscr{I}_{Y}(n) \longrightarrow \mathcal{O}_{P}(n) \longrightarrow \mathcal{O}_{Y}(n) \longrightarrow 0 \text {. }
$$

We get $H^{0}\left(\mathcal{O}_{P}(1)\right) \cong H^{0}\left(\mathcal{O}_{Y}(1)\right)$ and thus $H^{0}\left(\mathcal{O}_{P}(n)\right) \rightarrow H^{0}\left(\mathcal{O}_{Y}(n)\right)$ is surjective for all $n \geq 1$. The conditions on the $H^{\prime}$ 's now follow.

If $Y=Z(s)$ comes from a 2 bundle $E$ with $c_{1}=c_{2}=2$ then degree $Y$ $=2$ and $\omega_{Y}=\mathcal{O}_{Y}(-2)$. Our next step is to classify all such curves.

Proposition 1.2. Let $Y$ be a local complete intersection curve in $P$ of degree 2 and $\omega_{Y}=\mathcal{O}_{Y}(-2)$. Then $Y$ is either

i) 2 skew lines, or

ii) a nonreduced scheme structure on a line, given for example on the line $x=y=0$ by the homogeneous ideal $\left(x^{2}, x y, y^{2}, x F+y G\right)$ where $F$ and $G$ are linearly independent linear forms in $z$ and $w$.

Proof. See [5, Prop. 9.1(b)]. 
A curve satisfying ii) will be called a "double line".

Let $F$ be a coherent torsion free sheaf on $\boldsymbol{P}_{k}^{n}$ and let $P_{F}(n)=\chi(F(n)) / \mathrm{rank}$ $F$ where $\chi(F(n))$ is the Hilbert polynomial of $F$. Following Gieseker [3] we say $F$ is stable (respectively, semi-stable) if for all proper coherent subsheaves $G$ of $F, P_{G}(n)<P_{F}(n)$ (respectively, $P_{G}(n) \leq P_{F}(n)$ ). Inequality means $P_{G}(n)<P_{F}(n)$ for $n \gg 0$. Clearly (semi-)stability is independent of tensoring with $\mathcal{O}_{P_{k}^{n}}(t)$.

For a rank 2 vector bundle $E$ on $P$ with Chern classes $c_{1}$ and $c_{2}$ the Riemann Roch Theorem gives

$$
\chi(E)=\frac{1}{6}\left(\left(c_{1}^{3}-3 c_{1} c_{2}\right)+6\left(c_{1}^{2}-2 c_{2}\right)+11 c_{1}+12\right) .
$$

If $E(n)=E \otimes \mathcal{O}_{P}(n)$ then $c_{1}(E(n))=c_{1}+2 n$ and $c_{2}(E(n))=n^{2}+c_{1} n+c_{2}$. After twisting by a line bundle any rank 2 bundle on $P$ can be normalized so that $c_{1}=0$ or $c_{1}=-1$. We now give a criterion for stability.

Proposition 1.3. Let $E$ be a normalized rank 2 vector bundle on $P$ $=P_{k}^{3}$. Then $E$ is stable if and only if $H^{0}(P, E)=0$. If $E$ is not stable, then $E$ is semi-stable if and only if $E \simeq \mathcal{O}_{P} \oplus \mathcal{O}_{P}$.

Proof. To check (semi-)stability of a rank 2 bundle $E$ we need only to consider invertible subsheaves. Indeed, if $F$ is any rank 1 subsheaf of $E$ we may replace $F$ by a slightly larger sheaf if necessary (we denote this new subsheaf again by $F$ and its polynomial $P_{F}(n)$ is bigger than the original) and suppose that the quotient $Q=E / F$ is torsion free, rank 1. $Q$ is then isomorphic to $\mathscr{I}_{Y}(c)$ for some $c$ where $\mathscr{I}_{Y}$ is the ideal sheaf of a subscheme $Y$ of codimension $\geq 2$ or $Y$ is empty. If $Y$ is empty then $Q$ is invertible and then so is $F$. If codimension $Y \geq 2$ then since $\mathscr{I}_{Y}$ is locally generated by 2 elements (because it's a quotient of $E(-c)$ ) $Y$ is a local complete intersection curve. It follows that $\mathscr{I}_{Y}$ is locally generated by a regular sequence and has local homological dimension $1 . \quad F$ then has local homological dimension 0 so again is invertible.

Suppose $E$ is a normalized rank 2 bundle and $H^{\circ}(E)=0$. For any invertible subsheaf $F=\mathcal{O}_{P}(d)$ we have $c_{1}(F)=d<c_{1}(E) / 2=0$ or $-1 / 2$ and hence $E$ is stable. Let $E$ be a rank 2 bundle with Chern classes $c_{1}$ and $c_{2}$ and a global section $s$ such that $Y=Z(s)$ has codimension $=2$. The section $s$ defines an injection $\mathcal{O}_{P} \rightarrow E$. Comparing polynomials we get

$$
P_{E}(n)-P_{o_{p}}(n)=\frac{1}{3}\left(3 c_{1} n^{2}+3\left(c_{1}^{2}-2 c_{2}+6 c_{1}\right) n+c_{1}^{3}-3 c_{1} c_{2}+6\left(c_{1}^{2}-2 c_{2}\right)+11 c_{1}\right) .
$$


$P_{E}(n)-P_{o_{p}}(n) \geq 0$ exactly when $c_{1} \geq 0$. Furthermore if $c_{1}=0$ then $c_{2} \leq$ 0 . But $c_{2}=$ degree $Y \geq 0$. Suppose now that $E$ is stable and normalized then we have strict inequality and it follows that $H^{\circ}(E)=0$. If $E$ is semi-stable and we have equality then $E$ has a section $s$ with the degree of its zero set equal to 0, i.e. $s$ is nonvanishing and $E$ splits as $\mathcal{O}_{P} \oplus \mathcal{O}_{P}$.

Remark 1.3.1. The proof easily generalizes to give the same criterion for (semi-)stability of a rank 2 bundle on $\boldsymbol{P}_{k}^{n}, n \geq 2$.

Remark 1.3.2. There is an older definition of stability for vector bundles introduced by Mumford and Takemoto which is used in [1], [2] and [5]. This definition uses just the first Chern class instead of the entire Hilbert polynomial. The definition we use is the appropriate generalization to arbitrary coherent sheaves [7], [8]. The criterion we have obtained for stability of a rank 2 bundle on $\boldsymbol{P}_{k}^{n}$ is equivalent to the old definition, see [5, Section 3]. However for semi-stability of rank 2 bundles the old definition is less restrictive. In fact the restriction of a skew bundle to a general plane in $P$ is old semi-stable [5, Theorem 3.3] but it is not stable and is not decomposable (cf. the Chern classes) and so it is not new semistable.

We now consider skew bundles. Let $E$ be a skew bundle so $c_{1}=c_{2}$ $=2 . \quad E_{\text {norm }}=E(-1)$ has $c_{1}=0, c_{2}=1$. Let $s$ be a section of $E$ with $Y$ $=Z(s), 2$ skew lines. The sequence (1.0.1) becomes

$$
0 \longrightarrow \mathcal{O}_{P} \longrightarrow E \longrightarrow \mathscr{I}_{Y}(2) \longrightarrow 0 \text {. }
$$

From this we get $H^{0}(E(-1)) \simeq H^{0}\left(\mathscr{I}_{Y}(1)\right)=0$ since $Y$ does not lie in a plane. Thus $E$ is stable by our criterion.

Proposition 1.4. Skew bundles (up to twisting) are precisely the stable rank 2 bundles with $c_{1}=0, c_{2}=1$.

Proof. We must show that any such stable bundle $E$ is a skew bundle. By [5, Theorem 8.2] $H^{0}(E(1)) \neq 0$. Thus $E(1)$ has a global section $s$ such that $Y=Z(s)$ has codimension $=2$. Since $E(1)$ has $c_{1}=c_{2}=2$ we conclude from Proposition 1.2 that $Y$ is 2 skew lines or a double line. However, in Remark 3.3.4 we will see that any bundle corresponding to double lines is already a skew bundle.

The following result characterizes double coverings of $\boldsymbol{P}^{4}$ by the Grassmann variety of lines in $\boldsymbol{P}^{3}$. If $W$ is a 4 dimensional $k$-vector space then 
$G=\boldsymbol{G}_{2}\left(W^{\vee}\right)$ parametrizes 2-quotients of $W^{\vee}$ which are lines in $\boldsymbol{P}_{k}^{3}=\boldsymbol{P}\left(W^{\vee}\right)$. There is a closed immersion of $G$ into $\boldsymbol{P}_{k}^{5}=\boldsymbol{P}\left(\wedge^{2} W^{\vee}\right)$, the Plücker morphism. Let $V$ be a 5 dimensional vector space and $\boldsymbol{P}_{k}^{4}=\boldsymbol{P}\left(V^{\vee}\right)$.

Proposition 1.5. Let $f: G \rightarrow \boldsymbol{P}_{k}^{4}$ be a finite morphism of degree 2. Then there exists a closed point $m$ in $\boldsymbol{P}_{k}^{5}$ such that $f$ factors as the Plücker embedding followed by projection from the point $m$ and an automorphism of $\boldsymbol{P}_{k}^{4}$.

Proof. The Picard group of $G=G_{2}\left(W^{\vee}\right)$ is generated by the very ample invertible sheaf $\mathcal{O}_{G}(1)$ which gives the Plücker embedding $G \rightarrow \boldsymbol{P}_{k}^{5}$. The invertible sheaf $f^{*} \mathcal{O}_{p^{4}}(1)$ is isomorphic to $\mathcal{O}_{G}(n)$ for some $n>0$. The linear system defining $f, f^{*} H^{0}\left(\mathcal{O}_{P^{4}}(1)\right)$, is a subsystem of the complete linear system $H^{0}\left(\mathcal{O}_{G}(n)\right)$ which defines an embedding $g$ of degree $2 n^{5}$ of $G$ into some $\boldsymbol{P}^{N}$. The morphism $f$ factors as $\pi \circ g$ where $\pi$ is projection from a linear subspace in $\boldsymbol{P}^{N}$ onto $\boldsymbol{P}^{4}$ followed by an automorphism of $\boldsymbol{P}^{4}$. See $[4, I I, 7]$ for details about morphisms and linear systems. Since the degree of $f$ is the degree of $G$ in $P^{N}$ it follows that $n=1, N=5$ and the linear space is a closed point $m$ in $\boldsymbol{P}^{5}$.

Remark 1.5.1. Geometrically the fibres of the morphism $f$ are obtained by taking lines $L$ in $\boldsymbol{P}^{5}$ through $m$ and intersecting with $G$.

Remark 1.5.2. Given the morphism $f$ there is an intrinsic way of obtaining the point $m$. Pulling back the canonical 1-quotient sequence on $\boldsymbol{P}^{4}$ via $f$ gives a short exact sequence on $G$

$$
0 \longrightarrow K \longrightarrow V^{\vee} \otimes \mathcal{O}_{G} \longrightarrow \mathcal{O}_{G}(1) \longrightarrow 0 \text {. }
$$

$V^{\vee} \otimes \mathcal{O}_{G}$ is the trivial sheaf of rank 5 and $\mathcal{O}_{G}(1)$ is generated by five global sections. Taking global sections gives

$$
0 \longrightarrow V^{\vee} \longrightarrow H^{0}\left(\mathcal{O}_{G}(1)\right) \longrightarrow k \longrightarrow 0 \text {. }
$$

Making the canonical identifications $H^{0}\left(\mathcal{O}_{G}(1)\right) \simeq H^{0}\left(\mathcal{O}_{P^{5}}(1)\right) \simeq \wedge^{2} W^{\vee}$ we obtain the 1-quotient of $\wedge^{2} W^{\vee}$ corresponding to the point $m$.

We will make use of the theorem of Cohomology and Base Change. See [4, Theorem 12.11] for the statement and proof in the case of projective morphisms of noetherian schemes and scheme points. Since the results are local they extend to the case of local noetherian schemes. 
Passing from scheme fibres to geometric fibres is a faithfully flat base extension so it suffices to check the maps of cohomology on the geometric fibres.

Proposition 1.6. Let $f: X \rightarrow Y$ be a flat projective morphism of locally noetherian schemes such that for every geometric point $y$ in $Y, h^{0}\left(X_{y}, \mathcal{O}_{x_{y}}\right)$ $=1$ and $h^{1}\left(X_{y}, \mathcal{O}_{X_{y}}\right)=0$. Let $L$ and $M$ be invertible sheaves on $X$ such that $L_{y} \simeq M_{y}$ on $X_{y}$. Then $L \simeq M \otimes f^{*} N$ for some invertible sheaf $N$ on Y.

Proof. By taking the invertible sheaf $L \otimes M^{-1}$ we may suppose $M=$ $\mathcal{O}_{x}$ and must show that $L \simeq f^{*} N$. By Cohomology and Base Change it follows that $f_{*} L$ is invertible. If $\rho: f^{*} f_{*} L \rightarrow L$ is the canonical morphism then the restriction of $\rho$ to the geometric fibres is an isomorphism. It follows that $\rho$ is an isomorphism.

Our final result in this section is the following.

Proposition 1.7. Let $f, g: X \rightarrow Y$ be morphisms of $S$-schemes. Suppose $X$ is reduced and for every geometric point $s$ of $S$ the induced maps on the geometric fibres $X_{s} \rightarrow Y_{s}$ are the same. Then $f=g$.

Proof. It is easy to show that the underlying set maps are the same so we reduce to the case that $S, X$ and $Y$ are affine. Since the morphisms agree on the geometric fibres it follows they agree on the scheme fibres since this is a faithfully flat base extension. Finally we are reduced to the case of, say $C$-algebra homomorphisms, $\alpha, \beta: A \rightarrow B$ where $B$ is reduced and for every prime $P$ in $C$ the induced maps $A \otimes k(P) \rightarrow B \otimes k(P)$ agree. This means for all $x$ in $A \alpha(x)-\beta(x)$ is zero in $B \otimes k(P)$ for every prime $P$ in $C$. It follows that $\alpha(x)-\beta(x)=0$.

\section{§2. Families of skew bundles}

In this section we give a method of constructing families of skew bundles when the ground scheme $T$ is arbitrary. Applying this in the case $T=\operatorname{spec} Z$ we will obtain a family of skew bundles parametrized by a $Z$-scheme $M$ which is our candidate for the moduli.

From now on we fix a free $Z$-module $W$ of rank 4 . Projective three space is $P_{Z}^{3}=P\left(W^{\vee}\right)$ and $M=P\left(\wedge^{2} W^{\vee}\right)-G_{2}\left(W^{\vee}\right)$, the complement of the Grassmann in $P_{Z}^{5}$ under the Plücker embedding. Let $T$ be a scheme and $P=P\left(W_{T}^{\vee}\right)=P\left(W^{\vee}\right) \times{ }_{z} T$. On $P$ we have the canonical 1 -subbundle $0 \rightarrow$ 
$\mathcal{O}_{P}(-1) \rightarrow W_{P}=\mathcal{O}_{P} \otimes W$. Twisting once gives a section $s$ of $W_{P}(1)$. The Koszul Complex defined by $s$ is the exact sequence of trivial sheaves

$$
0 \longrightarrow \mathcal{O}_{P} \longrightarrow W_{P}(1) \longrightarrow \wedge^{2} W_{P}(2) \longrightarrow \wedge^{3} W_{P}(3) \longrightarrow \wedge^{4} W_{P}(4)=0 \text {. }
$$

After twisting by $\mathcal{O}_{P}(-2)$ let $\phi$ denote the map $\wedge^{2} W_{P} \rightarrow \wedge^{3} W_{P}(1)$. Let $K$ and $C$ denote the kernel and image respectively. We obtain a short exact sequence of locally free sheaves on $P$

$$
0 \longrightarrow K \longrightarrow \wedge^{2} W_{P} \stackrel{\phi}{\longrightarrow} C \longrightarrow 0
$$

Remark 2.0.2. If $\Omega_{P / T}$ is the sheaf of relative differentials we have [4, III, Example 8.4.b], the canonical exact sequence on $P$

$$
0 \longrightarrow \Omega_{P / T} \longrightarrow W_{P}^{\vee}(-1) \longrightarrow \mathcal{O}_{P} \longrightarrow 0
$$

Since $K$ is also the cokernel of $\mathcal{O}_{P}(-2) \rightarrow W_{P}(-1)$ it follows that $K$ is canonically isomorphic to $\Omega_{P / T}(2)^{\vee}$. If we choose a free basis for $W_{T}$ we can identify $\wedge^{4} W_{P} \simeq \mathcal{O}_{P}$ and the dual of the Koszul resolution becomes isomorphic to itself. In this way $C$ is non-canonically isomorphic to $\Omega_{P / T}(2)$.

Let $M_{T}=\boldsymbol{P}\left(\wedge^{2} W_{T}^{\vee}\right)-G_{2}\left(W_{T}^{\vee}\right)$. As an open subscheme of $\boldsymbol{P}\left(\wedge^{2} W_{T}^{\vee}\right), M_{T}$ inherits the canonical 1-subbundle sequence

$$
0 \longrightarrow \mathcal{O}_{M_{T}}(-1) \longrightarrow \wedge^{2} W_{M_{T}} \longrightarrow G \longrightarrow 0
$$

where $G$ is locally free of rank 5. Consider the following diagram of $T$ schemes

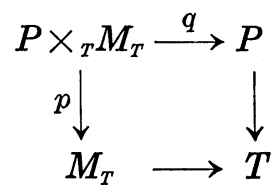

where $p$ and $q$ are the projections. On the product, which we denote simply by $P \times M$, we combine the pullbacks of (2.0.1) and (2.0.3) to obtain the following diagram 


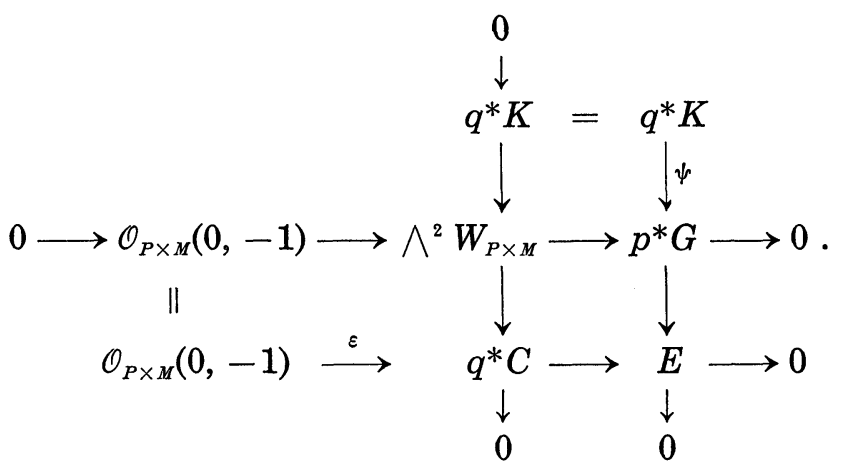

$\mathcal{O}_{P \times M}(0,-1)$ denotes the pullback of the invertible sheaf $\mathcal{O}_{M_{T}}(-1)$. The map $\psi$ is obtained by composition and $E=$ coker $\psi$ is a coherent sheaf. The bottom row is the induced surjection and $\varepsilon$ is composition. The construction of $E$ is functorial over the base $T$. That is, if $T^{\prime} \rightarrow T$ is a base change then the pullback of $E$ to the corresponding product scheme over $T^{\prime}$ is the cokernel of the same construction obtained by starting with the base scheme $T^{\prime}$ and the free module $W_{T^{\prime}}^{\vee}$. For any scheme $T$ we obtain a family $E$ of coherent sheaves on $\boldsymbol{P}_{T}^{3} \times_{T} M_{T}$ parametrized by $M_{T}$.

TheOREM 2.1. $E$ is a skew bundle parametrized by $M_{T}$.

Proof. We must show that the restriction of $E$ to the fibre over geometric points of $M_{T}$ is a skew bundle. We study these geometric fibres by taking a geometric point spec $k \rightarrow T$ and looking at the fibres over $k$-rational points of $M_{k}=M_{T} \times_{T}$ spec $k$.

We now are reduced to the construction in the case $T=\operatorname{spec} k, k$ an algebraically closed field. For a closed point $m$ in $M_{k}$ we identify the fibre $p^{-1}(m)$ with $P=\boldsymbol{P}\left(W_{k}^{v}\right)$. Restricting (2.0.4) to $p^{-1}(m)$ we obtain the following diagram.

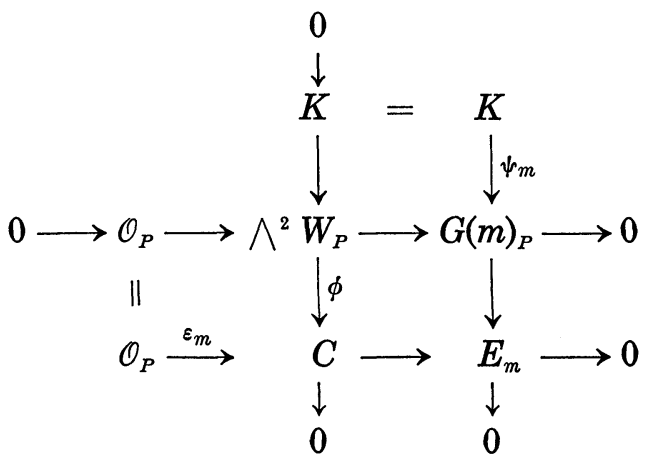


The middle row is a sequence of trivial sheaves and is the pullback from $k(m)=k$ of the sequence of vector spaces

$$
0 \longrightarrow k \longrightarrow \wedge^{2} W_{k} \longrightarrow G(m) \longrightarrow 0 \text {. }
$$

The image of $k$ is the 1-subspace spanned by an indecomposable vector (i.e. an element in $\wedge^{2} W_{k}$ not of the form $x \wedge y$ ) corresponding to the point $m$ in $M_{k}$.

We first show that $E_{m}$ is a rank 2 bundle. For a closed point $x$ in $P$ let $w_{x}$ be a generator of the 1-subspace in $W_{k}$ corresponding to $x$. At the fibre of (2.1.1) at $x$ the map $\phi(x)$ is given by $-\wedge w_{x}$ up to a scalar. $K(x)$ is the 3-subspace of $\wedge^{2} W_{k}$ consisting of decomposable vectors of the form $w \wedge w_{x}$. It follows that $\psi_{m}$ is an injection fibre by fibre of a rank 3 bundle on $P$ into a rank 5 bundle so the cokernel $E_{m}$ is a rank 2 bundle.

Both $\varepsilon_{m}$ and $\psi_{m}$ of (2.1.1) are injections and we get short exact sequences for the bottom row and right column. Since $K \simeq \Omega_{P / k}(2)^{\vee}$ cohomology of the right column of (2.1.1) gives an isomorphism of global sections $G(m)=$ $H^{0}\left(P, G(m)_{P}\right) \simeq H^{0}\left(P, E_{m}\right)$. We now show $E_{m}$ is a skew bundle. A nonzero section $s$ of $E_{m}$ gives a 1-subspace of $H^{0}\left(E_{m}\right)$ and hence a 1-subspace of $G(m)$. From (2.1.2) we see that this 1-subspace lifts to a 2-subspace $V$ of $\bigwedge^{2} W_{k}=H^{0}\left(\bigwedge^{2} W_{P}\right)$. For a closed point $x$ in $P$ we identify $V$ with its image in the fibre $\wedge^{2} W_{P} \otimes k(x) \simeq \bigwedge^{2} W_{k}$. Consider the fibre map of (2.1.1) $\alpha(x): \wedge^{2} W_{k} \rightarrow C(x) \rightarrow E_{m}(x)$. The image of $V$ under $\alpha(x)$ is zero exactly when the section $s$ vanishes at $x . \quad V$ corresponds to a line $L=\boldsymbol{P}\left(V^{v}\right)$ in $\boldsymbol{P}^{5}=\boldsymbol{P}\left(\bigwedge^{2} W_{k}^{\vee}\right)$ through $m$. Since the Grassmann $G=\boldsymbol{G}_{2}\left(W_{k}^{\vee}\right)$ is of degree 2 in $\boldsymbol{P}^{5} L$ meets $G$ in 2 points (or 1 point with multiplicity 2) corresponding to lines $\ell_{1}$ and $\ell_{2}$ in $P$. The image of $V$ under $\alpha(x)$ is zero exactly when $x$ lies on $\ell_{1} \cup \ell_{2}$, i.e. $s$ is zero precisely on $\ell_{1} \cup \ell_{2}$. We easily compute the Chern classes of $E_{m}$ to be $c_{1}=c_{2}=2$. It follows that the scheme zero set $Z(s)$ has degree 2 and $P_{a}=-1$ and so $Z(s)$ is two skew lines or a double line. The argument shows that every global section of $E_{m}$ determines a line in $\boldsymbol{P}^{5}$ through $m$. Conversely any line $L$ through $m$ determines a section $s$ of $E$ and $s$ vanishes on the lines in $P$ corresponding to the intersection of $L$ and $G$. Since there are always lines through $m$ meeting $G$ in 2 distinct points $E_{m}$ is a skew bundle.

Remark 2.1.3. From the bottom row of (2.1.1) we see we can view $E_{m}$ as a quotient of the rank 3 bundle $C \simeq \Omega_{P / k}(2)$ by a non-vanishing section. In Section 4 we extend our study to coherent sheaves which are quotients 
of $\Omega(2)$ by sections which vanish along a line.

Corollary 2.2. Let $E$ and $M_{T}$ be as in the previous theorem and let $p: P\left(W_{T}^{\vee}\right) \times_{T} M_{T} \rightarrow M_{T}$ be the projection. Then $\left(p_{*} E\right)^{\vee}$ is naturally isomorphic to the kernel of the canonical invertible quotient sequence on $M_{T}$

$$
0 \longrightarrow\left(p_{*} E\right)^{\vee} \longrightarrow \wedge^{2} W_{M_{T}}^{\vee} \longrightarrow \mathcal{O}_{M_{T}}(1) \longrightarrow 0 \text {. }
$$

Proof. From (2.0.4) we have the exact sequence $0 \rightarrow q^{*} K \rightarrow p^{*} G \rightarrow E$ $\rightarrow 0$. In (2.1.1) we studied the geometric fibre of this sequence and concluded that direct image cohomology gives $p_{*} p^{*} G=G \simeq p_{*} E$. The result now follows from (2.0.3).

Remark 2.2.2. The fibre of (2.2.1) at a geometric point $m$ of $M_{T}, k(m)$ $=k$, is

$$
0 \longrightarrow H^{0}\left(\boldsymbol{P}\left(W_{k}^{\vee}\right), E_{m}\right)^{\vee} \longrightarrow \wedge^{2} W_{k}^{\vee} \longrightarrow k \longrightarrow 0
$$

which is the dual of (2.1.2) and corresponds to $m$ as a closed point in $M_{k}$.

\section{§3. Representability of $S k$}

Let $T=\operatorname{spec} Z$ and let $\mathscr{E}$ be the skew bundle of Theorem 2.1 which is parametrized by the open subscheme $M=\boldsymbol{P}\left(\bigwedge^{2} W^{\vee}\right)-\boldsymbol{G}_{2}\left(W^{\vee}\right)$ in $\boldsymbol{P}_{Z}^{5} \cdot \mathscr{E}^{\circ}$ defines in a natural way a morphism of functors $\Phi: \operatorname{Hom}(, M) \rightarrow S k$. We now show that $\Phi$ is an isomorphism of functors and thus the pair $(M, \mathscr{E})$, or more accurately $M$ and the class of $\mathscr{E}$, represent the functor $S k$.

For the time being let $E$ be a skew bundle over an algebraically closed field $k$ and let $P=P\left(W_{k}^{v}\right)$. Suppose $s$ is a global section of $E$ such that $Y=Z(s)$ is 2 skew lines. The section determines the exact sequence (1.0.1) which becomes

$$
0 \longrightarrow \mathcal{O}_{P} \longrightarrow E \longrightarrow \mathscr{I}_{Y}(2) \longrightarrow 0
$$

In Proposition 1.1 it was shown that the homogeneous ideal of $Y$ is generated by 4 quadrics. Thus the sheaf $\mathscr{I}_{Y}(2)$ is generated by global sections and $h^{\circ}\left(P, \mathscr{I}_{Y}(2)\right)=4$. It follows easily from (3.0.1) that $E$ is generated by global sections and $h^{0}(P, E)=5$. Twisting (3.0.1) by $n$ and taking cohomology gives an isomorphism $H^{1}(P, E(n)) \simeq H^{1}\left(P, \mathscr{I}_{Y}(n+2)\right)$. Again from Proposition 1.1 we conclude that $H^{1}(P, E(n))=0$ for $n \neq-2$ and $H^{1}(P, E(-2))$ $=k$. Also $H^{0}(P, E(-1)) \simeq H^{0}\left(P, \mathscr{I}_{Y}(1)\right)=0$ since $Y$ does not lie in a plane.

Let $L$ be a line in $P$. Restriction of $E$ to $L$ gives an exact sequence 


$$
\left.0 \longrightarrow \mathscr{I}_{L} \cdot E \longrightarrow E \longrightarrow E\right|_{L} \longrightarrow 0 .
$$

LEMMA 3.1. Cohomology of (3.0.2) gives the exact sequence

$$
0 \longrightarrow H^{0}\left(P, \mathscr{I}_{L} \cdot E\right) \longrightarrow H^{0}(P, E) \longrightarrow H^{0}\left(P,\left.E\right|_{L}\right) \longrightarrow 0 .
$$

Furthermore $h^{0}\left(\mathscr{I}_{L} \cdot E\right)=1$ and $h^{0}\left(\left.E\right|_{L}\right)=4$.

Proof. The line $L$ is a complete intersection in $P$ so $\mathscr{I}_{L}$ has a standard locally free resolution which we tensor with $E$ to obtain

$$
0 \longrightarrow E(-2) \longrightarrow \stackrel{2}{\oplus} E(-1) \longrightarrow \mathscr{I}_{L} \cdot E \longrightarrow 0 \text {. }
$$

We see that $H^{1}\left(P, \mathscr{I}_{L} \cdot E\right)$ injects into $H^{2}(P, E(-2))$ which is Serre dual to $H^{1}\left(P, E^{\vee}(2)(-4)\right)=H^{1}(P, E(-4))=0$. This gives exactness of (3.1.1) on the right. The above sequence also gives $H^{0}\left(P, \mathscr{I}_{L} \cdot E\right) \simeq H^{1}(P, E(-2))=k$. The rest of the lemma follows easily.

Lemma 3.2. For every line $L$ in $P=P\left(W_{k}^{\vee}\right)$ there exists a section of $E$ unique up to a scalar multiple vanishing on $L$.

Proof. $H^{0}\left(P, \mathscr{I}_{I}, E\right)$ is the subspace of global sections of $E$ vanishing on $L$ and has dimension one.

We now fix a (locally noetherian) scheme $T$ and a skew bundle $E$ parametrized by $T$. Let $P=P\left(W_{T}^{\vee}\right), G=G_{2}\left(W_{T}^{\vee}\right)$ and $\pi$ and $\rho$ be the respective structure morphisms as $T$-schemes. Let $F \subset P \times_{T} G$ be the flag scheme. We have the following commutative diagram of $T$-schemes with projections $\sigma$ and $\tau$

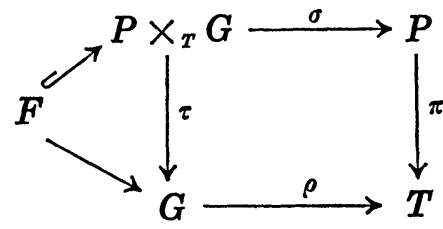

One shows easily that $\pi_{*} E$ is locally free of rank 5 and the fibre at a geometric point $t$ of $T$ is $\pi_{*} E(t) \simeq H^{0}\left(P_{t}, E_{t}\right)$. Let $P\left(\left(\pi_{*} E\right)^{v}\right)$ be the projective bundle over $T$ with geometric fibre over $t$ equal to $P\left(H^{0}\left(P_{t}, E_{t}\right)^{v}\right)$.

Proposition 3.3. Given $T$ and $E$ as above then there exists a morphism of T-schemes

$$
f: G \longrightarrow P\left(\left(\pi_{*} E\right)^{\vee}\right)
$$

such that for a geometric point $t$ of $T$ the fibre map $f_{t}$ takes a closed point 
of $G_{t}$ corresponding to a line $L$ in $P_{t}$ to the point corresponding to the 1quotient $H^{0}\left(P_{t}, \mathscr{I}_{L} \cdot E_{t}\right)^{\nu}$ of $H^{0}\left(P_{t}, E_{t}\right)^{\nu}$. That is, $f_{t}$ maps the line $L$ to the essentially unique section of $E_{t}$ vanishing on $L$.

Proof. The subscheme $F$ in $P \times_{T} G$ satisfies the usual exact sequence which we tensor with $\sigma^{*} E$ to obtain

$$
0 \longrightarrow \mathscr{I}_{F} \cdot \sigma^{*} E \longrightarrow \sigma^{*} E \longrightarrow \mathcal{O}_{F} \otimes \sigma^{*} E \longrightarrow 0 \text {. }
$$

To take direct image cohomology of this sequence we study the geometric fibres of $\tau$ by taking a geometric point $t$ of $T$ and looking at the fibres of $\tau_{t}$ over closed points $\ell$ in $G_{t}$. The restriction of (3.3.1) to the fibre $\tau_{t}^{-1}(\ell) \simeq P_{t}$ is (3.0.2) for the bundle $E_{t}$ where $\ell$ corresponds to the line $L$ in $P_{t}$. Using cohomology and base change it is straight forward to show that cohomology of (3.3.1) gives the exact sequence

$$
0 \longrightarrow \tau_{*}\left(\mathscr{I}_{F} \cdot \sigma^{*} E\right) \longrightarrow \tau_{*} \sigma^{*} E \longrightarrow \tau_{*}\left(\mathcal{O}_{F} \otimes \sigma^{*} E\right) \longrightarrow 0
$$

of locally free sheaves of rank 1,5 and 4 respectively. The fibre of this sequence at $\ell$ in $G_{t}$ is (3.1.1) for the bundle $E_{t}$. Identifying $\tau_{*} \sigma^{*} E \simeq \rho^{*} \pi_{*} E$ and dualizing gives an invertible quotient on $G$

$$
0 \longrightarrow \tau_{*}\left(\mathcal{O}_{F} \otimes \sigma^{*} E\right)^{\vee} \longrightarrow \rho^{*}\left(\left(\pi_{*} E\right)^{\vee}\right) \longrightarrow \tau_{*}\left(\mathscr{I}_{F} \cdot \sigma^{*} E\right)^{\vee} \longrightarrow 0 .
$$

Clearly this invertible quotient defines the desired morphism.

Remark 3.3.3. In the above situation the geometric fibre map $f_{t}$ is a 2-1 covering since every section of $E_{t}$ vanishes on 2 lines or 1 line with multiplicity 2 (by Proposition 1.2 these are the only curves that can come from a skew bundle). From Proposition 1.5 we get a closed point $m_{t}$ in $\boldsymbol{P}\left(\wedge^{2} W_{k(t)}^{\vee}\right)$ which gives a bijection between the set of lines $L$ through $m_{t}$ and points in $P\left(H^{0}\left(P_{t}, E_{t}\right)^{v}\right)$. The associated section vanishes on the lines in $P_{t}$ corresponding to the intersection of $L$ and $G_{t}$. We call $m_{t}$ the "point of the bundle $E_{t}$ ". Since some lines through $m_{t}$ meet $G_{t}$ with multiplicity 2 it is apparent that every skew bundle $E_{t}$ has sections vanishing on double lines.

Remark 3.3.4. Let $Y$ be a double line of the type in Proposition 1.2 and let $Y_{\text {red }}=L \simeq \boldsymbol{P}^{1}$ be the reduced line. It turns out (see the referenced proof of the proposition) that $\mathscr{I}_{Y}$ is the kernel of a surjective map $\mathscr{I}_{L} \rightarrow$ $\mathcal{O}_{L}$. Using this exact sequence one obtains immediately the same results as in Proposition 1.1 for the case of a double line. The same arguments 
now give Lemmas 3.1 and 3.2 for bundles $E$ corresponding to double lines. We again get a 2-1 covering $f$ of $\boldsymbol{P}\left(H^{0}(E)^{\vee}\right)$ by the Grassmann of lines and the fibres of $f$ correspond to the lines through a closed point in $\boldsymbol{P}\left(\bigwedge^{2} W_{k}^{\vee}\right)$. It now follows that $E$ also has sections vanishing on 2 skew lines. Thus double lines do not give any new bundles.

We now give our key technical result.

Theorem 3.4. Let $E$ be a skew bundle parametrized by $T$. Then there exists an equivalent bundle $E^{\prime}$ such that $\left(\pi_{*} E^{\prime}\right)^{\vee}$ is the kernel of an invertible quotient map

$$
0 \longrightarrow\left(\pi_{*} E^{\prime}\right)^{\vee} \longrightarrow \wedge^{2} W_{T}^{\vee} \longrightarrow \mathscr{L} \longrightarrow 0 \text {. }
$$

The quotient defines a morphism of Z-schemes $g: T \rightarrow M \subset P\left(\bigwedge^{2} W^{\vee}\right)$ which satisfies the property $(*)$ for $E^{\prime}$.

(*) If $z$ is a geometric point of $\operatorname{spec} Z, k(z)=k$, then the fibre map $g_{z}$ takes a $k$-rational point $t$ of $T_{z}$ to $m_{t}$ in $\boldsymbol{P}\left(\bigwedge^{2} W_{k}^{v}\right)$ where $m_{t}$ is the point of $E_{t}^{\prime}$ of Remark 3.3.3.

Furthermore if $E^{\sim}$ is another skew bundle such that $\left(\pi_{*} E^{\sim}\right)^{\vee}$ defines the same morphism $g$ and $g$ satisfies $(*)$ for $E^{\sim}$ then $E^{\sim} \simeq E^{\prime}$.

Proof. We begin with the invertible quotient (3.3.2). For a geometric point $t$ of $T, k(t)=k$, the restriction of (3.3.2) to $G_{t}$ gives the invertible quotient which defines $f_{t}$. In Proposition 1.5 we saw that this invertible quotient is isomorphic to $\mathcal{O}_{G_{t}}(1)$. By Proposition 1.6 the invertible quotient of (3.3.2) is isomorphic to $\mathcal{O}_{G}(1) \otimes \rho^{*} L$ where $L$ is a line bundle on $T$ and $\mathcal{O}_{G}(1)$ defines the Plücker embedding of $G$.

We replace $E$ by the equivalent bundle $\pi^{*} L^{-1} \otimes E$ which we again denote by $E$ and (3.3.2) becomes

$$
0 \longrightarrow \tau_{*}\left(\mathcal{O}_{F} \otimes \sigma^{*} E\right)^{\vee} \longrightarrow \rho^{*}\left(\left(\pi_{*} E\right)^{\vee}\right) \longrightarrow \mathcal{O}_{G}(1) \longrightarrow 0 \text {. }
$$

Changing $E$ by $\pi^{*} L^{-1}$ changes $P\left(\left(\pi_{*} E\right)^{\vee}\right)$ to $P\left(L \otimes\left(\pi_{*} E\right)^{\vee}\right)$ but they are canonically isomorphic so we still have the "same" morphism $f$. The restriction of the above sequence to the geometric fibre $G_{t}$ gives the exact sequence which defines $f_{t}$,

$$
0 \longrightarrow\left(\tau_{*}\left(\mathcal{O}_{F} \otimes \sigma^{*} E\right)^{v}\right)_{t} \longrightarrow H^{0}\left(P_{t}, E_{t}\right)^{\vee} \otimes G_{t} \longrightarrow \mathcal{O}_{G_{t}}(1) \longrightarrow 0 .
$$

In Remark 1.5.2 we saw that the cohomology of this sequence is 


$$
0 \longrightarrow H^{0}\left(P_{t}, E_{t}\right)^{\nu} \longrightarrow \wedge^{2} W_{k}^{\vee} \longrightarrow k \longrightarrow 0,
$$

and the 1-quotient corresponds to the point $m_{t}$ of the bundle $E_{t}$. It now follows that direct image cohomology of (3.4.1) gives on $T$

$$
0 \longrightarrow\left(\pi_{*} E\right)^{\vee} \longrightarrow \wedge^{2} W_{T}^{\vee} \longrightarrow R^{1} \rho_{*}\left(\tau_{*}\left(\mathcal{O}_{F} \otimes \sigma^{*} E\right)^{\vee}\right) \longrightarrow 0 \text {. }
$$

We have made the canonical identification $\rho_{*} \mathcal{O}_{G}(1) \simeq \Lambda^{2} W_{T}^{\vee}$ which comes from the Plücker embedding of $G$ in $\boldsymbol{P}\left(\wedge^{2} W_{T}^{\vee}\right)$.

The fibre of this sequence at a geometric point $t$ is (3.4.2) and $R^{1} \rho_{*}\left(\tau_{*}\left(\mathcal{O}_{F} \otimes \sigma^{*} E\right)^{\vee}\right)$ is an invertible quotient which defines the morphism $g$ which satisfies $(*)$.

Suppose now that $E^{\sim}$ is another skew bundle such that $\left(\pi_{*} E^{\sim}\right)^{\nu}$ defines the same morphism $g$ satisfying $(*)$. This says that there is an isomorphism $\left(\pi_{*} E^{\sim}\right)^{\vee} \simeq\left(\pi_{*} E\right)^{\vee}$ such that for each geometric point $t$ of $T$ the induced isomorphism $H^{0}\left(P_{t}, E_{t}^{\sim}\right) \simeq H^{0}\left(P_{t}, E_{t}\right)$ preserves zero sets of the sections since both bundles have the same point $m_{t}$. We noted that a skew bundle is generated by global sections so the canonical morphism $\pi^{*} \pi_{*} E \rightarrow E$ is surjective and similarly for $E^{\sim}$. If $K$ and $K^{\sim}$ denote respectively the kernels then the above isomorphism gives on $P$,

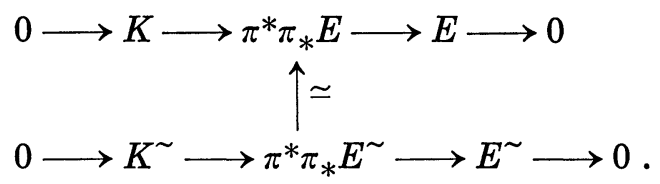

One concludes easily by looking at the geometric fibres that $K^{\sim} \simeq K$ and so $E^{\sim} \simeq E$. This completes the proof of the theorem.

The theorem says that given a skew bundle $E$ parametrized by $T$ then in the equivalence class of $E$ there is a distinguished element $E^{\prime}$ which defines the morphism $g$ with property $(*)$.

We now determine the morphism $g$ for the skew bundle $\mathscr{E}$ parametrized by the open scheme $M$ in $P_{Z}^{5}$.

Let $p: \boldsymbol{P}\left(W_{M}^{\vee}\right) \rightarrow M$ be the projection. The invertible quotient sequence (2.2.1) defines the morphism $\mathrm{id}_{m}$. The fibre of this sequence at a geometric point $m$ of $M, k(m)=k$, is (2.2.3). Clearly $m$ is the point of the bundle $\mathscr{E}_{m}$. This means that if $g$ is the morphism of the previous theorem then for a geometric point $z$ of $\operatorname{spec} Z$ the fibre maps $g_{z}, \mathrm{id}_{M_{z}}: M_{z} \rightarrow M_{z}$ agree on $k(z)$-rational points and hence agree on the geometric fibre $M_{z}$ since $M_{z}$ is reduced and of finite type over $k(z)$. Now by Proposition $1.7 \mathrm{~g}$ must 
be $\mathrm{id}_{M}$. Thus $\mathscr{E}$ is the distinguished element in its class and the morphism it defines is $\mathrm{id}_{M}$.

TheOREM 3.5. The morphism of functors $\Phi: \operatorname{Hom}(, M) \rightarrow$ Sk defined by $\mathscr{E}$ is an isomorphism.

Proof. Let $T$ be a locally noetherian scheme. We must show that the set map $\Phi(T): \operatorname{Hom}(T, M) \rightarrow S k(T)$ is a bijection. Let $E$ be the distinguished representative of its class in $S k(T)$ which defines the morphism $g: T \rightarrow M$ of Theorem 3.4. We have the following diagram

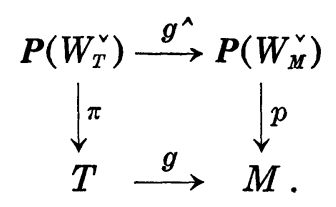

The morphisms $\pi$ and $p$ are the canonical ones and $g^{\wedge}$ is the map induced by $g$. Let $E^{\wedge}=g^{\wedge} \mathscr{E}$, which is a skew bundle. The construction in Theorem 3.4 is functorial; i.e., each sequence in the construction for $E^{\wedge}$ is the pullback of the corresponding sequence for $\mathscr{E}$. Thus the morphism $g_{E^{\wedge}}: T \rightarrow M$ determined by $E^{\wedge}$ is $g$ followed by the morphism determined by $\mathscr{E}$ which is $\mathrm{id}_{M}$. Hence $g_{E^{\wedge}}=g$ and by the uniqueness property of Theorem 3.4 $E^{\wedge}$ is isomorphic to $E$. We have shown that $\Phi(T)$ is surjective.

Suppose $g: T \rightarrow M$. Let $E=g^{\wedge} * \mathscr{E}$. We have just seen that $g_{E}=g$ so it follows that $\Phi(T)$ is injective. This completes the proof.

Remark 3.5.1. If $T=\operatorname{spec} k, k$ an algebraically closed field, then the map $\Phi(T)$ gives a bijection between Hom ( $\left.\operatorname{spec} k, M_{k}\right)=$ closed points of $M_{k}$ and the isomorphism classes of skew bundles on $\boldsymbol{P}\left(W_{k}^{v}\right)$. Any automorphism of $\boldsymbol{P}\left(W_{k}^{\vee}\right)$ is induced by an automorphism of the vector space $W_{k}^{\vee}$ which in turn induces an automorphism of $\Lambda^{2} W_{k}^{v}$. Thus we get a group homomorphism Aut $\boldsymbol{P}\left(W_{k}^{\vee}\right) \rightarrow$ Aut $\boldsymbol{P}\left(\bigwedge^{2} W_{k}^{\vee}\right)$ with $\sigma \mapsto \bigwedge^{2} \sigma$. It is easy to verify by choosing a basis of $W_{k}^{v}$ that there are exactly two orbits of the closed points of $\boldsymbol{P}\left(\bigwedge^{2} W_{k}^{\vee}\right)$ under this action, namely $\boldsymbol{G}_{2}\left(W_{k}^{\vee}\right)$ and the complement $M_{k}$. The correspondence between bundles and points is preserved under the action in the sense that if $E$ is a skew bundle with point $m$ then $\sigma^{*} E$ is the skew bundle with point $\wedge^{2} \sigma^{-1}(m)$. Since the action is transitive on $M_{k}$ any two skew bundles are related by an automorphism of $\boldsymbol{P}\left(W_{k}^{v}\right)$. 


\section{§4. Stability}

The construction of the skew bundle $\mathscr{E}$ parametrized by the open subscheme $M$ extends to give a coherent sheaf parametrized by $\boldsymbol{P}_{Z}^{5}$. Since $\boldsymbol{P}_{Z}^{5}$ is the natural projective closure of $M$ it is reasonable to investigate the sheaves we get by restriction to the fibres over geometric points of $\boldsymbol{P}_{Z}^{5}$.

We modify the construction in Section 2 as follows. For a scheme $T$ we replace $M_{T}$ by $\boldsymbol{P}_{T}^{\delta}=\boldsymbol{P}\left(\bigwedge^{2} W_{T}^{\vee}\right)$ and obtain a diagram similar to (2.0.4) which again defines the sheaf $E=$ coker $\psi$. We study the restriction to the fibres over geometric points of $\boldsymbol{P}_{T}^{5}$ by the usual trick of reducing to the case $T=\operatorname{spec} k, k$ an algebraically closed field, and considering the restriction to the fibres over $k$-rational points of $\boldsymbol{P}_{k}^{b}=\boldsymbol{P}\left(\bigwedge^{2} W_{k}^{\vee}\right)$.

From now on we fix the scheme $\boldsymbol{T}=\operatorname{spec} k$ and $\boldsymbol{P}=\boldsymbol{P}_{k}^{3}=\boldsymbol{P}\left(W_{k}^{\vee}\right)$. The restriction of (2.0.4) to the fibre $p^{-1}(\ell) \simeq P, \ell$ a closed point of $G=G_{2}\left(W_{k}^{v}\right)$, gives the same diagram (2.1.1) (replace $m$ by $\ell$ ) and (2.1.2) now becomes

$$
0 \longrightarrow k \longrightarrow \wedge^{2} W_{k} \longrightarrow G(\ell) \longrightarrow 0 \text {. }
$$

The image of $k$ is now spanned by a decomposable vector corresponding to the point $\ell$ in $G$. Let $L$ be the line in $P$ corresponding to $\ell$. Using the same analysis as we did in Section 2 it is easy to show that the map $K \rightarrow G(\ell)_{P}$ as in (2.1.1) is injective at the fibres except at points in $L . E_{\ell}$ is a coherent sheaf of rank 2 except at points in $L$ where the rank jumps to 3. Again we get a bijection between the set of lines in $\boldsymbol{P}_{k}^{b}$ through $\ell$ and sections of $H^{\circ}\left(E_{\ell}\right)$ upto scalar multiple. In Remark 2.1.3 we viewed a skew bundle as a quotient of $\Omega(2)=\Omega_{P / k}(2)$ by a nonvanishing section. In the present case we can view $E_{\ell}$ as a quotient of $\Omega(2)$ by a section which vanishes on $L . \quad E_{\ell}$ is torsion free since the section does not vanish along a divisor.

LEMma 4.1. If a section $s$ of $\Omega(2)$ vanishes on a line $L$ then $Z(s)=L$, i.e. $s$ vanishes with multiplicity 1.

Proof. Suppose $L$ has equations $X_{0}=X_{1}=0$ in $P$. $\Omega(2)$ fits into the exact sequence

$$
0 \longrightarrow \Omega(2) \longrightarrow \stackrel{4}{\oplus} \mathcal{O}_{P}(1) \longrightarrow \mathcal{O}_{P}(2) \longrightarrow 0
$$

and

$$
H^{0}(\Omega(2))=\operatorname{ker}\left(\stackrel{4}{\oplus} H^{0}\left(\mathcal{O}_{P}(1)\right) \longrightarrow H^{0}\left(\mathcal{O}_{P}(2)\right)\right)
$$


where the map is given by $X_{i} d X_{j} \mapsto X_{i} X_{j}$ see [4, II, Theorem 8.13] and the proof. The $d X_{i}$ 's denote the basis. $H^{0}(\Omega(2))$ has a basis $X_{i} d X_{j}-X_{j} d X_{i}$ for $0 \leq i<j \leq 3$ and $s$ is given by $X_{0} d X_{1}-X_{1} d X_{0}$. The local equations for $s$ are $X_{0}=X_{1}=0$ and so $Z(s)=L$.

The techniques used in Proposition 1.3 for determining stability of a rank 2 bundle fail to go through for an arbitrary coherent sheaf. To check the stability of the coherent sheaves $E_{\ell}$ we use an ad hoc method. First we establish several technical results.

4.1.1. Let $A$ be a noetherian local ring and $M$ a finitely generated $A$-module with a finite projective resolution. Let pd $M$ denote the projective dimension of $M$. We have the following inequality [9, Section 16, Example 4] pd $M+\operatorname{depth} M=\operatorname{depth} A$.

4.1.2. Let $A$ be regular local ring and $M$ a finitely generated $A$-module. Then $\operatorname{pd} M \leq r$ if and only if $\operatorname{Ext}^{i}(M, A)=0$ for all $i>r$, see [4, III, Example 6.6].

LEMMA 4.2. Let $F$ be a coherent sheaf on $P$. Then $\mathscr{E}_{x t_{P}^{i}}\left(F^{\vee}, \mathcal{O}_{P}\right)=0$ for all $i>1$.

Proof. Choose a locally free sheaf $L$ such that $0 \rightarrow K \rightarrow L \rightarrow F \rightarrow 0$. Dualizing we obtain

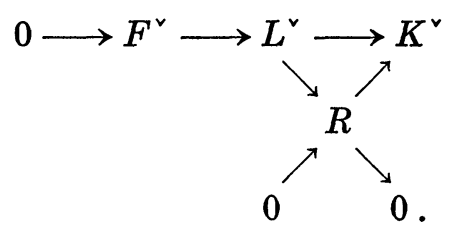

From the short exact sequence we get $\mathscr{E}_{x t_{P}^{i}}\left(F^{\vee}, \mathcal{O}_{P}\right) \simeq \mathscr{E} x t_{P}^{i+1}\left(R, \mathcal{O}_{P}\right)$ for $i>0$. Since $R$ injects into the torsion free sheaf $K$ we have depth $R_{x} \geq 1$ for all $x$ in $P$. From 4.1.1 and 4.1.2 we get $\mathscr{E}_{x t_{P}^{i+1}}\left(R, \mathcal{O}_{P}\right)=0$ for $i>1$ which proves the lemma.

4.2.1. Let $Y$ be a closed subscheme of codimension $r$ in $P$. Then $\mathscr{E} x t_{P}^{i}\left(\mathcal{O}_{Y}, \mathcal{O}_{P}\right)=0$ for $i<r$. See [4, III, Lemma 7.3].

4.2.2. Let $\mathscr{I}_{Y}$ be a sheaf of ideals where $Y$ has codimension $Y \geq 2$ in $P$. Then dualizing the standard exact sequence $0 \rightarrow \mathscr{I}_{Y} \rightarrow \mathcal{O}_{P} \rightarrow \mathcal{O}_{Y} \rightarrow 0$ and using 4.2.1 we get $\mathscr{I}_{Y}^{v} \simeq \mathcal{O}_{P}$. 
Let $\ell$ be a closed point of $G$ corresponding the line $L$ in $P$ and let $E_{\ell}$ be the coherent sheaf we constructed with the point $\ell . \quad E_{\ell}$ embeds into its double dual $E_{\ell}^{\nu \nu}$. Recall that $\omega_{L}=\mathscr{E} x t_{P}^{2}\left(\mathcal{O}_{L}, \omega_{P}\right) \simeq \mathcal{O}_{L}(-2)$ and $\omega_{P} \simeq \mathcal{O}_{P}(-4)$.

Proposition 4.3. $E_{\ell}^{\vee \vee} \simeq \mathcal{O}_{P}(1) \oplus \mathcal{O}_{P}(1)$.

Proof. The first step is to show that $E_{\ell}^{v v}$ is locally free. $E_{\ell}$ is the quotient of $\Omega(2)$ by a section $s$ which gives the exact sequence

$$
0 \longrightarrow \mathcal{O}_{P} \longrightarrow \Omega(2) \longrightarrow E_{\ell} \longrightarrow 0 \text {. }
$$

From Lemma $4.2, Z(s)=L$. Dualizing the sequence we get

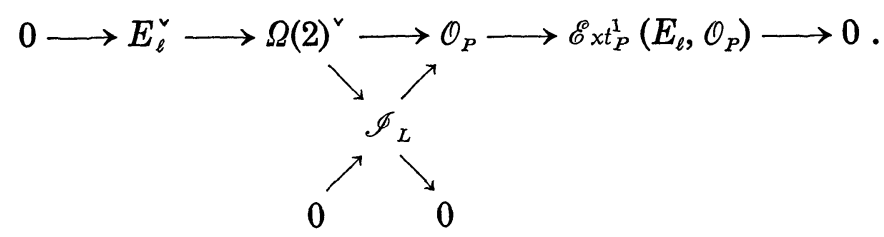

Hence $\mathscr{E} x t_{P}^{1}\left(E_{\ell}, \mathcal{O}_{P}\right) \simeq \mathcal{O}_{L}$. Dualizing the first short exact sequence we obtain

$$
0 \longrightarrow \mathscr{I}_{L}^{v} \longrightarrow \Omega(2) \longrightarrow E_{\ell}^{v v} \longrightarrow \mathscr{E}^{2} t_{P}^{1}\left(\mathscr{I}_{L}, \mathcal{O}_{P}\right) \longrightarrow 0 .
$$

We have $\mathscr{I}_{L}^{\vee} \simeq \mathcal{O}_{P}$ by 4.2 .2 .

From the exact sequence

$$
0 \longrightarrow \mathscr{I}_{L} \longrightarrow \mathcal{O}_{P} \longrightarrow \mathcal{O}_{L} \longrightarrow 0 \text {. }
$$

We get $\mathscr{E}_{x t_{P}^{1}}\left(\mathscr{I}_{L}, \mathcal{O}_{P}\right) \simeq \mathscr{E}_{x t_{P}^{2}}\left(\mathcal{O}_{L}, \mathcal{O}_{P}\right) \simeq \omega_{L}(4) \simeq \mathcal{O}_{L}(2)$.

Combining the exact sequences we get

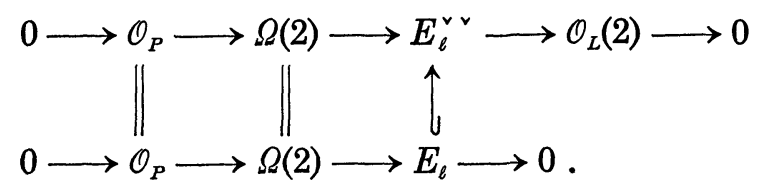

It follows that

$$
0 \longrightarrow E_{\ell} \longrightarrow E_{\ell}^{\vee \vee} \longrightarrow \mathcal{O}_{L}(2) \longrightarrow 0 \text {. }
$$

Again, applying $\mathscr{H}_{\mathrm{om}}\left(, \mathcal{O}_{P}\right)$ to this exact sequence and using 4.2.1 we get

$$
\begin{aligned}
0 \longrightarrow \mathscr{E}_{x t_{P}}^{1}\left(E_{\ell}^{\vee v}, \mathcal{O}_{P}\right) \longrightarrow \mathscr{E}^{0} x t_{P}^{1}\left(E_{\ell}, \mathcal{O}_{P}\right) \longrightarrow \mathscr{E}^{2} t_{P}^{2}\left(\mathcal{O}_{L}(2), \mathcal{O}_{P}\right) & \longrightarrow \mathscr{E} x t_{P}^{2}\left(E_{\ell}^{v \vee}, \mathcal{O}_{P}\right) .
\end{aligned}
$$

$\mathscr{E} x t_{P}^{2}\left(E_{\ell}^{\vee v}, \mathcal{O}_{P}\right)=0$ by Lemma 4.2. The middle two terms are both isomorphic 
to $\mathcal{O}_{L}$, hence $\mathscr{E} x t_{P}^{1}\left(E_{\ell}^{\vee \vee}, \mathcal{O}_{P}\right)=0$ and by 4.1 .2 . it follows that $E_{\ell}^{\vee \vee}$ is locally free.

From (4.3.1) we get

$$
H^{1}\left(E_{\ell}(n)\right) \simeq H^{1}(\Omega(2+n))=\left\{\begin{array}{l}
0, n \neq-2 \\
k, n=-2 .
\end{array}\right.
$$

and $c_{1}\left(E_{\ell}\right)=c_{2}\left(E_{\ell}\right)=2$. From (4.3.2) we compute $c_{1}\left(E_{\ell}^{v v}\right)=2$ and $c_{2}\left(E_{\ell}^{v v}\right)=1$. Since $H^{0}\left(E_{\ell}(-1)\right)=0$ we have $H^{0}\left(E_{\ell}^{\vee v}(-3)\right)=0$. We claim that $H^{0}\left(E_{\ell}^{\vee v}(-2)\right)$ $=0$. Otherwise, $E_{\ell}^{\vee v}(-2)$ has a section $s$ with $Y=Z(s)$ of codimension 2 , degree $Y=1$ and $\omega_{Y}=\mathcal{O}_{Y}(-6)$ which is impossible. Since $H^{1}\left(E_{\ell}(-1)\right)=0$ we get $H^{0}\left(E_{\ell}^{\vee v}(-1)\right) \simeq H^{0}\left(\mathcal{O}_{L}(1)\right)$. Hence $E_{\ell}^{\vee v}(-1)$ has a section $s$ with degree $Z(s)=0$ and thus $s$ is nonvanishing. The bundle $E_{\ell}^{v v}(-1)$ splits as $\mathcal{O}_{P} \oplus \mathcal{O}_{P}$. This proves the proposition.

We now give our result on the stability of the $E_{\ell}$ 's.

TheOREM 4.4. For closed points $\ell$ in $G$ the coherent sheaves $E_{\ell}$ are stable sheaves with the same Chern classes and hence Hilbert polynomial as skew bundles.

Proof. Let $L$ be the line in $P$ corresponding to $\ell$. We have just shown that the $E_{\ell}$ 's have the same Chern classes as skew bundles. Let $F$ be a rank 1 subsheaf of $E_{\ell}$ with a torsion free quotient $Q$. Then $F \simeq \mathscr{I}_{z}(c)$ and $Q \simeq \mathscr{I}_{W}(d)$ where $Z$ and $W$ have codimension $\geq 2$ or are empty. By 4.2.2 $\mathscr{I}_{Z}(c)^{\nu \vee} \simeq \mathcal{O}_{P}(c)$. But $\mathscr{I}_{Z}(c)^{\nu \vee} \longrightarrow E_{\ell}^{\nu v} \simeq \mathcal{O}_{P}(1) \oplus \mathcal{O}_{P}(1)$ so $c \leq 1$. If $c<1$ then $P_{F}(n)<P_{E_{\ell}}(n)$ since the leading coefficient of $P_{E_{\ell}}(n)-P_{F}(n)$ is $c_{1}\left(E_{\ell}\right) / 2$ $-c=1-c>0$. Suppose $c=1$. Since $H^{0}\left(E_{\ell}(-1)\right)=0$ we have $\mathscr{I}_{z}(1) \neq$ $\mathcal{O}_{P}(1)$. We have the following exact diagram

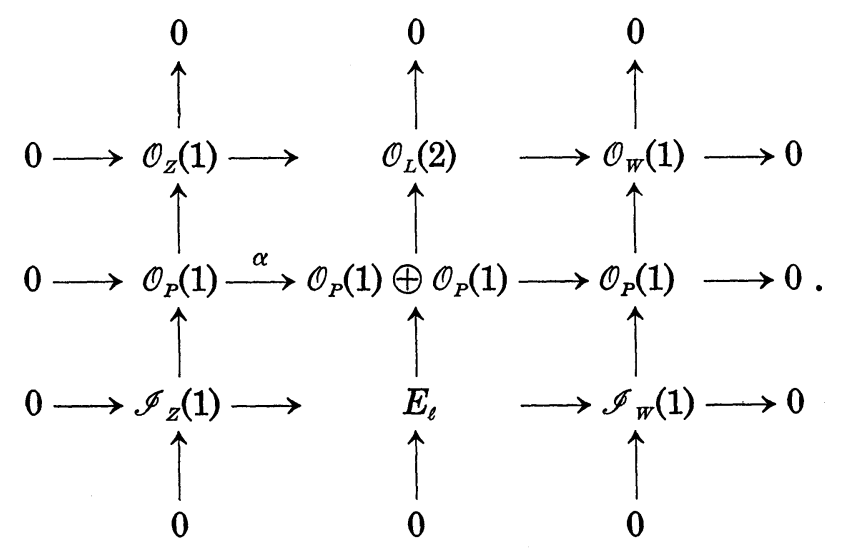


Clearly support $Z=L$ and $Z$ is reduced so $Z=L$ and $W$ is a reduced point. Thus we consider subsheaves $0 \rightarrow \mathscr{I}_{L}(1) \rightarrow E_{\ell}$ which exist, viz. image $(\alpha) \cap$ $E_{\ell}$ in $E_{\ell}^{\vee v}$. Computing polynomials we get $P_{E_{\ell}}(n)-P_{L}(n+1)=(n+1) / 2$ $>0$. We conclude that $E_{\ell}$ is a stable sheaf.

The family of coherent sheaves $E_{p}, p$ a closed point in $\boldsymbol{P}_{K}^{5}$, consists of the quotients of $\Omega(2)$ by global sections. In (1.3.3) we saw that skew bundles occur as extensions. This is also true for the sheaves $E_{\ell}$. Indeed, let $E_{\ell}$ be a quotient of $\Omega(2)$ by a section $t$ which vanishes on the line $L$. Recall that there is a bijection between lines $S$ in $P^{5}$ through $\ell$ and $\left(H^{\circ}\left(P, E_{l}\right)-\right.$ $0) / k^{*}$. Let $S$ be a line through $\ell$ which meets $G$ in exactly one other point $\ell^{\prime}$ corresponding to $L^{\prime}$ in $P$. Let $s^{\prime}$ be the section of $E_{\ell}$ corresponding to $S$ and let $Q$ be the quotient $E_{\ell} / s^{\prime} \cdot \mathcal{O}_{P}$. The section $s^{\prime}$ lifts to a section $t^{\prime}$ of $\Omega(2)$ and we get

$$
0 \longrightarrow \stackrel{2}{\oplus} \mathcal{O}_{P} \longrightarrow \Omega(2) \longrightarrow Q \longrightarrow 0 .
$$

The span of $t, t^{\prime}$ in $H^{\circ}(\Omega(2))$ contains a nonvanishing section $t^{\prime \prime}$ which gives a skew bundle $E$ and $Q$ is a quotient of $E$ by a section vanishing on the skew lines $Y=L \cup L^{\prime}$. Hence $Q \simeq \mathscr{I}_{Y}(2)$ and we obtain $E_{\ell}$ as an element of $\operatorname{Ext}_{P}^{1}\left(\mathscr{I}_{Y}(2), \mathcal{O}_{P}\right)$.

Proposition 4.5. The family of sheaves $E_{P}, p$ a closed point in $\boldsymbol{P}_{k}^{5}$, consists of precisely the sheaves which occur as elements of $\operatorname{Ext}_{P}^{1}\left(\mathscr{I}_{Y}(2), \mathcal{O}_{P}\right)$ for all possible pairs of skew lines $Y$ in $\boldsymbol{P}_{k}^{3}$.

Proof. We have just observed that every $E_{p}$ is such an extension. Conversely if $Y=L \cup L^{\prime}$ is a pair of skew lines let $\ell, \ell^{\prime}$ be the corresponding points on the Grassmann $G$. From the exact sequence

$$
0 \longrightarrow \mathscr{I}_{Y}(2) \longrightarrow \mathcal{O}_{P}(2) \longrightarrow \mathcal{O}_{Y}(2) \longrightarrow 0
$$

we get $\operatorname{Ext}_{P}^{1}\left(\mathscr{I}_{Y}(2), \mathcal{O}_{P}\right) \simeq \operatorname{Ext}_{P}^{2}\left(\mathcal{O}_{Y}(2), \mathcal{O}_{P}\right)$. The spectral sequence of local and global Ext degenerates, since $Y$ is a local complete intersection, to give $\operatorname{Ext}_{P}^{2}\left(\mathcal{O}_{Y}(2), \mathcal{O}_{P}\right) \simeq H^{0}\left(P, \mathscr{E} x t_{P}^{2}\left(\mathcal{O}_{Y}(2), \mathcal{O}_{P}\right)\right) \simeq H^{0}\left(Y, \omega_{Y}(2)\right) \simeq H^{0}\left(Y, \mathcal{O}_{Y}\right)=k \oplus k$. If $S$ is the line in $P^{5}$ through $\ell$ and $\ell^{\prime}$ then each point $p$ on $S$ gives a sheaf $E_{p}$ with a section $s$ upto scalar such that $E_{p} / s \cdot \mathscr{O}_{P} \simeq \mathscr{I}_{Y}(2)$, i.e. an element of $\operatorname{Ext}_{p}^{1}\left(\mathscr{I}_{Y}(2), \mathcal{O}_{P}\right) / k^{*}=\boldsymbol{P}_{k}^{1}$. Conversely any element of $\operatorname{Ext}_{p}^{1}\left(\mathscr{I}_{Y}(2), \mathcal{O}_{P}\right)$ which corresponds to a unit in $H^{0}\left(\mathcal{O}_{Y}\right)$ determines an extension

$$
0 \longrightarrow \mathcal{O}_{P} \longrightarrow E \longrightarrow \mathscr{I}_{Y}(2) \longrightarrow 0
$$


where $E$ is a rank 2 bundle, hence a skew bundle (see proof of [5, Theorem 1.1]). Thus we have a (noncanonical) bijection between points of $S$ and elements in $\operatorname{Ext}_{P}^{1}\left(\mathscr{I}_{Y}(2), \mathcal{O}_{P}\right) / k^{*}$.

The writer has been unable to determine if there are any other stable or semi-stable coherent sheaves on $\boldsymbol{P}_{k}^{3}$ with the same Hilbert polynomial as the skew bundles.

\section{BIBLIOGRAPHY}

[1] Barth, W., Some properties of stable rank 2 vector bundles on $\boldsymbol{P}_{n}$, Math. Ann., 226 (1977), 125-150.

[2] — Moduli of vector bundles on the projective plane, Invent. Math., 42 (1977), $63-91$.

[ 3 ] Gieseker, D., On the moduli of vector bundles on an algebraic surface, Annals of Math., 106 (1977), 45-60.

[4] Hartshorne, R., Algebraic Geometry, Graduate Texts in Math., 52, SpringerVerlag, Heidelberg, New York, 1977.

[ 5 ] - Stable vector bundles of rank 2 on $P^{3}$, Math. Ann., 235 (1978), 229-280.

[6] Horrocks, G., Vector bundles on the punctured spectrum of a local ring, Proc. Lond. Math. Soc., (3), 14 (1964), 689-713.

[ 7 ] Maruyama, M., Moduli of stable sheaves, I, J. Math., Kyoto Univ., 17 (1977), 91-126.

[ 8 ] - Moduli of stable sheaves, II, J. Math. Kyoto Univ., 18 (1978).

[ 9 ] Matsumura, H., Commutative Algebra, W.A. Benjamin Co., New York, 1970.

Department of Mathematics

The University of Kansas

Lawrence, Kansas 66045

$U S A$ 